\title{
The Retrotrapezoid Nucleus and Breathing
}

\author{
Patrice G. Guyenet, Ruth L. Stornetta, Stephen B.G. Abbott, Seth D. Depuy, and Roy \\ Kanbar \\ Department of Pharmacology, University of Virginia, 1300 Jefferson Park Avenue, 800735, \\ Charlottesville, VA 22908-0735, USA \\ Patrice G. Guyenet: pgg@virginia.edu
}

\begin{abstract}
The retrotrapezoid nucleus (RTN) is located in the rostral medulla oblongata close to the ventral surface and consists of a bilateral cluster of glutamatergic neurons that are non-aminergic and express homeodomain transcription factor Phox $2 \mathrm{~b}$ throughout life. These neurons respond vigorously to increases in local $\mathrm{pCO}_{2}$ via cell-autonomous and paracrine (glial) mechanisms and receive additional chemosensory information from the carotid bodies. RTN neurons exclusively innervate the regions of the brainstem that contain the respiratory pattern generator (RPG). Lesion or inhibition of RTN neurons largely attenuates the respiratory chemoreflex of adult rats whereas their activation increases respiratory rate, inspiratory amplitude and active expiration. Phox $2 b$ mutations that cause congenital central hypoventilation syndrome in humans prevent the development of RTN neurons in mice. Selective deletion of the RTN Phox2b-VGLUT2 neurons by genetic means in mice eliminates the respiratory chemoreflex in neonates.

In short, RTN Phox2b-VGLUT2 neurons are a major nodal point of the CNS network that regulates $\mathrm{pCO}_{2}$ via breathing and these cells are probable central chemoreceptors.
\end{abstract}

\section{Keywords}

Breathing network; Chemoreflexes; Central chemoreceptors; Retrotrapezoid nucleus; Congenital central hypoventilation syndrome; Phox2b; Optogenetics

\subsection{Introduction}

The central respiratory chemoreflex is the stimulation of breathing caused by an increase in

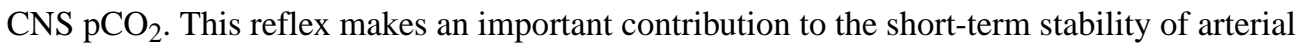
$\mathrm{pCO}_{2}$. It provides an especially powerful respiratory stimulus during apnea, airway obstruction and deliberate or accidental inhalation of $\mathrm{CO}_{2}$. The central respiratory chemoreflex never operates in isolation since $\mathrm{CO}_{2}$ also controls breathing via the carotid bodies. This essential interaction is discussed elsewhere (Blain et al. 2010).

The central chemoreflex is presumably triggered by the interaction of protons or, possibly, molecular $\mathrm{CO}_{2}$ (Huckstepp et al. 2010) with unidentified sensors ((Guyenet et al. 2010) for 
review). As explained below, the retrotrapezoid nucleus (RTN) is a critical nodal point of the CNS network responsible for the central respiratory chemoreflex and this nucleus seems to derive much of its chemosensitivity from a local effect of $\mathrm{CO}_{2}$ although the exact location of the proton receptors (RTN neurons, glia or both) is unsettled. The existence of other putative CNS chemoreceptors is discussed elsewhere (Nattie and Li 2009; Corcoran et al. 2009;

Guyenet et al. 2010).

\subsection{The Retrotrapezoid Nucleus: Definition and Chemosensitivity}

The name RTN was coined in 1989 to designate a group of superficial neurons that are located under the facial motor nucleus and innervate the ventral respiratory column (Smith et al. 1989). This thin sheet of cells extends rostrally up to the caudal end of the trapezoid body, hence the name. Smith et al. (1989) speculated that these neurons could have a chemosensory role because their location roughly coincides with a previously identified chemosensitive region of the ventral medullary surface of the cat. Nattie and his colleagues were able to produce reliable albeit modest increases in ventilation in rats $\left(\sim 25 \% \mathrm{~V}_{\mathrm{E}}\right)$ by artificially acidifying the RTN region (Li and Nattie 2002). These authors produced comparable ventilatory effects by acidifying many other brainstem regions and, on this basis, they concluded that RTN was one among many brain regions that contain central respiratory chemoreceptors ( $\mathrm{Li}$ and Nattie 2002). This viewpoint is developed elsewhere (see, for example Nattie and Li 2009; Corcoran et al. 2009).

The RTN neurons that regulate breathing remained unidentified for a long time for lack of a histological marker and because this "nucleus" is in reality a sparse collection of cells that are not clearly demarcated from adjoining portions of the reticular formation. Recently, the RTN region was shown to contain a distinctive group of non-aminergic $\mathrm{CO}_{2}$-sensitive neurons that express VGLUT2 and the transcription factor Phox $2 b$, henceforth called the RTN Phox2b-VGLUT2 neurons (Mulkey et al. 2004; Stornetta et al. 2006). These findings were seminal in two respects. They provided a histological marker for the putative chemoreceptors. The presence of Phox $2 \mathrm{~b}$ enabled investigators to introduce foreign proteins such as channelrhodopsin-2 or the allatostatin receptor relatively specifically into these neurons using lentiviral vectors engineered with PRSx8, a high-efficiency Phox2-activated artificial promoter (Hwang et al. 2001; Abbott et al. 2009). Figure 16.1 depicts the location and projections of the $\sim 2,000$ RTN Phox2b-VGLUT2 neurons in the rat.

\subsection{How Do RTN Neurons Respond to $\mathrm{CO}_{2}$ ?}

RTN neurons are very sensitive to hypercapnia ( $0.5 \mathrm{~Hz}$ per 0.01 unit change in pHa), $0.5 \mathrm{~Hz}$ representing approximately 5\% of their entire dynamic range (Guyenet et al. 2005) (Fig. 16.2a). This high $\mathrm{CO}_{2}$ sensitivity may result from several mechanisms (Fig. 16.3). The level of evidence supporting each mechanism varies.

Evidence for a cell autonomous pH-sensitivity (Fig. 16.3, mechanism 1) comes from observations that RTN neurons are excited by $\mathrm{CO}_{2}$ or acidification in slices and that this response persists in the presence of blockers of GABA, glutamate and ATP receptors (Mulkey et al. 2004; Lazarenko et al. 2009) (Fig. 16.2b). This work, performed in neonatal 
brain slices, has certain limitations. For example, the $\mathrm{pH}$ sensitivity of the neurons is only $40 \%$ of that observed in vivo, even when temperature is factored in (Guyenet et al. 2005) (Fig. 16.2a, b). This may be because this work was performed using tissue from neonate rodents which have weaker chemoreflexes. However, the most glaring hole in the theory that RTN neurons are cell-autonomous $\mathrm{CO}_{2}$ sensors is that the proton receptor(s) is(are) unidentified (Guyenet et al. 2010).

Evidence for a contribution of surrounding glial cells to the $\mathrm{pH}$ response of RTN neurons (Fig. 16.3, mechanism 2) derives from two main observations. The glial cells of the ventral medullary surface are depolarized by acid and, like astrocytes elsewhere, this glia releases ATP (Gourine et al. 2010; Mulkey and Wenker 2011). Secondly, photostimulation of Channelrhodopsin2-transfected RTN glial cells increases breathing, an effect that is attenuated by administration of an ATP receptor antagonist (Gourine et al. 2010). This evidence also has its limitations. A $\leq \mathrm{kb}$ segment of the GFAP promoter was used to drive expression of ChR2 into the glia using an adenovirus. Short promoters are rarely totally cellspecific. Secondly, the respiratory effects produced by activating glial cells seems too persistent ( $\sim 30 \mathrm{~min}$ ) to elicit timely corrective respiratory adjustments to $\mathrm{CO}_{2}$ changes (Gourine et al. 2010). Finally, blocking ATP receptors has only small effects on the response of RTN neurons to acidification in slices (Mulkey et al. 2004; Wenker et al. 2010).

The exquisite sensitivity of RTN neurons to $\mathrm{CO}_{2}$ in vivo could also be due to the presence of a local circuit (gap junctions, recurrent excitatory collaterals) that amplifies their cellautonomous response to $\mathrm{pH}$ (Fig. 16.3, mechanism 3). During the prenatal period, RTN neurons are coupled by gap junctions that contribute to their group pacemaker activity (Thoby-Brisson et al. 2009).

RTN neurons could also be a convergence point for additional central chemoreceptors, orexin and serotonin being two such candidates (Nattie and Li 2009; Corcoran et al. 2009) (Fig. 16.3, mechanism 4). This hypothesis is still controversial (Depuy et al. 2011) and needs further testing.

The fifth mechanism is a polysynaptic input from the peripheral chemoreceptors (Fig. 16.3, mechanism 5). This input is well-documented and involves a glutamatergic projection from the caudal portion of the nucleus solitary tract to the RTN (Takakura et al. 2006).

\subsection{Contribution of the RTN Neurons to the Chemoreflexes}

In adult rats, RTN Phox2b-VGLUT2 neurons mediate a large portion of the central respiratory chemoreflex. Selective photoactivation of RTN Phox2b-VGLUT2 neurons transfected with Channelrhodopsin2 produces a massive stimulation of breathing (Kanbar et al. 2010). Relatively selective lesions of the RTN neurons attenuate the central chemoreflex of anesthetized rats according to the degree of cell loss (Takakura et al. 2008). Finally, selective inhibition of the RTN neurons in conscious rats with intact carotid bodies attenuates the response to $\mathrm{CO}_{2}$ by $60 \%$ on average (Marina et al. 2010).

Mice with genetic deletion of RTN Phox2b-VGLUT2 neurons lack central respiratory chemosensitivity at birth (Goridis and Brunet 2010). The first generation of these 
experiments involved making a heterozygous mouse that uniformly expresses the toxic (27 alanine) mutation of Phox $2 b$ (Dubreuil et al. 2008). Mice carrying this mutation have no RTN and die within hours of birth from respiratory failure and possibly other undetected deficits. Subsequently, Phox $2 b$ was selectively excised from subsets of neurons that depend, like the RTN, on a particular set of transcription factors such as KROX20 (EGR2) or ATOH-1 (Dubreuil et al. 2009). Each manipulation aborted the development of the RTN Phox2b-VGLUT2 neurons and produced mice that failed to breathe after birth. In all these models, the core of the respiratory pattern generator developed seemingly normally but lacked $\mathrm{CO}_{2}$ sensitivity in vitro revealing that the respiratory network of the neonate is insensitive to moderate acidification ( $0.2 \mathrm{pH}$ units) in the absence of RTN neurons. This important observation seems to eliminate the possibility that $\mathrm{pH}$-sensitivity is more or less ubiquitous within the respiratory network. The main interpretative limitation of this data is that other neurons besides the RTN Phox2b-VGLUT2 neurons could have been eliminated or impaired by these genetic crosses. In a recent study from the same group, the toxic 27 alanine mutation was selectively introduced in Egr2-dependent neurons resulting in an even more specific deletion of the RTN neurons than in prior experiments (Ramanantsoa et al. 2011). These mice have a selective, complete loss of the RTN Phox2b-VGlut2 neurons, but survive into adulthood despite having no central respiratory chemoreflex up to 2 weeks after birth.

In summary, the respiratory chemoreflex is entirely dependent on the integrity of the RTN Phox2b-VGLUT2 neurons at and shortly after birth. Remarkably, selective genetic deletion of the RTN Phox2b-VGLUT2 neurons and loss of breathing stimulation by $\mathrm{CO}_{2}$ is survivable, apparently because of a compensatory, possibly hypoxic, drive from the carotid bodies (Ramanantsoa et al. 2011). In adulthood at least $60 \%$ of the chemoreflex depends on the RTN.

\subsection{How Do the RTN Neurons Regulate the Respiratory Network and What Else Do They Control?}

RTN Phox2b-VGLUT2 neurons activate the breathing rate, increase the respiratory rhythmic contractions of the diaphragm and activate abdominal expiratory muscles (Janczewski and Feldman 2006; Abdala et al. 2009b; Marina et al. 2010; Kanbar et al. 2010). Further understanding of how these various outflows are controlled will require deciphering the connections of RTN Phox2b-VGLUT2 neurons. This difficult integrative issue has only received partial answers to date. RTN neurons innervate the entire ventral respiratory group, suggesting that they target many types of respiratory neurons (Abbott et al. 2009). The preBötzinger complex is among these targets and RTN neurons likely regulate the respiratory rate at this level (Bochorishvili et al. 2011). RTN also innervates the caudal most part of the respiratory column which contains expiratory premotor neurons (Iscoe 1998; Abbott et al. 2009). This projection likely underpins some of the regulation of active (phase-2) expiration by RTN neurons but more rostral portions of the ventral respiratory column also contribute to this response (Pagliardini et al. 2011). The respiratory network includes many inhibitory neurons that would not be active without an excitatory drive (Smith et al. 2009). These neurons are also plausible targets of the RTN. 
There may be several subgroups of RTN Phox2b-VGLUT2 neurons. For example, only twothirds of the RTN Phox2b-VGLUT2 neurons are Atoh-1 dependent for their development in mice (Ramanantsoa et al. 2011). Functional heterogeneity between the Phox2b-VGLUT2 neurons is also suggested by the fact that, in neonatal rat brainstem maintained in vitro, these neurons are either phasically (preI-post-I neurons) or tonically active (Onimaru et al. 2008). Both types respond to $\mathrm{CO}_{2}$ however, and their differences in discharge patterns may result from the fact that these neurons are in the process of losing their embryonic group pacemaker properties (Thoby-Brisson et al. 2009). In the adult, RTN Phox2b-VGLUT2 neurons have a variety of stereotyped respiratory modulations (Guyenet et al. 2005) that denote differences in input and, possibly, functional heterogeneity. Finally, the RTN region contains neurons whose activity is restricted to the late expiratory period and become active only at very high levels of $\mathrm{CO}_{2}$ (Abdala et al. 2009a). These neurons could conceivably be specialized in driving active expiration but evidence that they are Phox2b-VGLUT2 neurons is currently lacking.

Finally, based on their projections, RTN Phox2b-VGLUT2 neurons could regulate other notably $\mathrm{CO}_{2}$-dependent $\mathrm{CNS}$ outflows such as airway tone, $\mathrm{CO}_{2}$-induced arousal, the stimulatory effect of $\mathrm{CO}_{2}$ on cardiovascular function, and the urge to breathe.

\subsection{Pathophysiology}

Non-catecholaminergic Phox2b-positive neurons most likely homologous to the rodent RTN have been identified in man (Rudzinski and Kapur 2010). CCHS (congenital central hypoventilation syndrome) is a developmental disease whose signs and symptoms include attenuated or absent chemoreflexes, sleep apnea and loss of the sensation of dyspnea (Weese-Mayer et al. 2010). Expansion of the 20-alanine tract of Phox $2 \mathrm{~b}$ is the most frequent cause of this syndrome (Weese-Mayer et al. 2010). These mutations produce a dominant negative and toxic version of the transcription factor which, in mice, impairs the development or survival of RTN neurons with considerable specificity as explained above (Dubreuil et al. 2008; Goridis and Brunet 2010). The loss of the RTN has yet to be documented in CCHS. It is a plausible cause of the respiratory deficits experienced by these patients but is unlikely to account for the entire syndrome (Weese-Mayer et al. 2010). The development of the carotid bodies, sympathetic post-ganglionic neurons and the nucleus of the solitary tract are all Phox2b-dependent (Goridis and Brunet 2010). Developmental abnormalities in any of these regions could interfere with autonomic regulations and exacerbate the chemoreflex deficit caused by RTN loss.

\subsection{Conclusions}

The RTN Phox2b-VGLUT2 neurons are a major nodal point through which lung ventilation is regulated by $\mathrm{CO}_{2}$. Evidence that these neurons are central respiratory chemoreceptors is considerable but will remain incomplete until the sensor(s) for $\mathrm{pH}\left(\right.$ or $\left.\mathrm{pCO}_{2}\right)$ are identified and proof is obtained that the RTN Phox2b-VGLUT2 neurons respond to $\mathrm{pCO}_{2}$ in a cell autonomous manner. At the present time, the $\mathrm{CO}_{2}$ sensitivity of the RTN neurons is best explained by a mixture of cell-autonomous and paracrine responses to $\mathrm{pH} / \mathrm{pCO}_{2}$. 
A superficial analogy between a sensory organ like the carotid body and the RTN is tempting but one should not lose sight of the fact that the RTN Phox2b-VGLUT2 neurons receive synaptic inputs from multiple brain regions (e.g. hypothalamus, the respiratory network and the raphe) and are subject to regulation by polysynaptic inputs from cardiopulmonary receptors.

The RTN Phox2b-VGLUT2 neurons enhance the breathing rate, inspiratory amplitude and active expiration. Subsets of RTN Phox2b-VGLUT2 neurons may mediate the orderly recruitment of various muscle groups when the organism is exposed to incremental levels of $\mathrm{CO}_{2}$. Finally, the carotid body input to RTN neurons underscores the highly integrated nature of central and peripheral chemoreflexes.

\section{Supplementary Material}

Refer to Web version on PubMed Central for supplementary material.

\section{References}

Abbott SBG, Stornetta RL, Fortuna MG, Depuy SD, West GH, Harris TE, Guyenet PG. Photostimulation of retrotrapezoid nucleus Phox $2 \mathrm{~b}$-expressing neurons in vivo produces longlasting activation of breathing in rats. J Neurosci. 2009; 29:5806-5819. [PubMed: 19420248]

Abdala AP, Rybak IA, Smith JC, Paton JF. Abdominal expiratory activity in the rat brainstem-spinal cord in situ: patterns, origins, and implications for respiratory rhythm generation. J Physiol. 2009a; 587:3539-3559. [PubMed: 19491247]

Abdala AP, Rybak IA, Smith JC, Zoccal DB, Machado BH, St-John WM, Paton JF. Multiple pontomedullary mechanisms of respiratory rhythmogenesis. Respir Physiol Neurobiol. 2009b; 168:19-25. [PubMed: 19540366]

Blain GM, Smith CA, Henderson KS, Dempsey JA. Peripheral chemoreceptors determine the respiratory sensitivity of central chemoreceptors to CO(2). J Physiol. 2010; 588:2455-2471. [PubMed: 20421288]

Bochorishvili G, Stornetta RL, Coates MB, Guyenet PG. Pre-Bötzinger complex receives glutamatergic innervation from galaninergic and other retrotrapezoid nucleus neurons. J Comp Neurol. 2011; 520(5):1047-1061.

Corcoran AE, Hodges MR, Wu Y, Wang W, Wylie CJ, Deneris ES, Richerson GB. Medullary serotonin neurons and central CO2 chemoreception. Respir Physiol Neurobiol. 2009; 168:49-58. [PubMed: 19394450]

Depuy SD, Kanbar R, Coates MB, Stornetta RL, Guyenet PG. Control of breathing by raphe obscurus serotonergic neurons in mice. J Neurosci. 2011; 31:1981-1990. [PubMed: 21307236]

Dubreuil V, Ramanantsoa N, Trochet D, Vaubourg V, Amiel J, Gallego J, Brunet JF, Goridis C. A human mutation in Phox $2 \mathrm{~b}$ causes lack of $\mathrm{CO} 2$ chemosensitivity, fatal central apnoea and specific loss of parafacial neurons. Proc Natl Acad Sci U S A. 2008; 105:1067-1072. [PubMed: 18198276]

Dubreuil V, Thoby-Brisson M, Rallu M, Persson K, Pattyn A, Birchmeier C, Brunet JF, Fortin G, Goridis C. Defective respiratory rhythmogenesis and loss of central chemosensitivity in phox $2 b$ mutants targeting retrotrapezoid nucleus neurons. J Neurosci. 2009; 29:14836-14846. [PubMed: 19940179]

Fortin G, Thoby-Brisson M. Embryonic emergence of the respiratory rhythm generator. Respir Physiol Neurobiol. 2009; 168:86-91. [PubMed: 19560563]

Goridis C, Brunet JF. Central chemoreception: lessons from mouse and human genetics. Respir Physiol Neurobiol. 2010; 173:312-321. [PubMed: 20307691]

Gourine AV, Kasymov V, Marina N, Tang F, Figueiredo MF, Lane S, Teschemacher AG, Spyer KM, Deisseroth K, Kasparov S. Astrocytes control breathing through pH-dependent release of ATP. Science. 2010; 329:571-575. [PubMed: 20647426] 
Guyenet PG, Mulkey DK, Stornetta RL, Bayliss DA. Regulation of ventral surface chemoreceptors by the central respiratory pattern generator. J Neurosci. 2005; 25:8938-8947. [PubMed: 16192384]

Guyenet PG, Stornetta RL, Bayliss DA. Central respiratory chemoreception. J Comp Neurol. 2010; 518:3883-3906. [PubMed: 20737591]

Huckstepp RT, Id BR, Eason R, Spyer KM, Dicke N, Willecke K, Marina N, Gourine AV, Dale N. Connexin hemichannel-mediated CO2-dependent release of ATP in the medulla oblongata contributes to central respiratory chemosensitivity. J Physiol. 2010; 588:3901-3920. [PubMed: 20736421]

Hwang DY, Carlezon WA Jr, Isacson O, Kim KS. A high-efficiency synthetic promoter that drives transgene expression selectively in noradrenergic neurons. Hum Gene Ther. 2001; 12:1731-1740. [PubMed: 11560767]

Iscoe S. Control of abdominal muscles. Prog Neurobiol. 1998; 56:433-506. [PubMed: 9775401]

Janczewski WA, Feldman JL. Distinct rhythm generators for inspiration and expiration in the juvenile rat. J Physiol. 2006; 570:407-420. [PubMed: 16293645]

Kanbar R, Stornetta RL, Cash DR, Lewis SJ, Guyenet PG. Photostimulation of Phox2b medullary neurons activates cardiorespiratory function in conscious rats. Am J Respir Crit Care Med. 2010; 182:1184-1194. [PubMed: 20622037]

Lazarenko RM, Milner TA, Depuy SD, Stornetta RL, West GH, Kievits JA, Bayliss DA, Guyenet PG. Acid sensitivity and ultrastructure of the retrotrapezoid nucleus in Phox2b-EGFP transgenic mice. J Comp Neurol. 2009; 517:69-86. [PubMed: 19711410]

Li A, Nattie E. CO2 dialysis in one chemoreceptor site, the RTN: stimulus intensity and sensitivity in the awake rat. Respir Physiol Neurobiol. 2002; 133:11-22. [PubMed: 12385727]

Marina N, Abdala AP, Trapp S, Li A, Nattie EE, Hewinson J, Smith JC, Paton JF, Gourine AV. Essential role of Phox $2 \mathrm{~b}$-expressing ventrolateral brainstem neurons in the chemosensory control of inspiration and expiration. J Neurosci. 2010; 30:12466-12473. [PubMed: 20844141]

Mulkey DK, Wenker IC. Astrocyte chemoreceptors: mechanisms of $\mathrm{H}+$ sensing by astrocytes in the retrotrapezoid nucleus and their possible contribution to respiratory drive. Exp Physiol. 2011; 96:400-406. [PubMed: 21169332]

Mulkey DK, Stornetta RL, Weston MC, Simmons JR, Parker A, Bayliss DA, Guyenet PG. Respiratory control by ventral surface chemoreceptor neurons in rats. Nat Neurosci. 2004; 7:1360-1369. [PubMed: 15558061]

Nattie E, Li A. Central chemoreception is a complex system function that involves multiple brain stem sites. J Appl Physiol. 2009; 106:1464-1466. [PubMed: 18467549]

Onimaru H, Ikeda K, Kawakami K. CO2-sensitive preinspiratory neurons of the parafacial respiratory group express Phox2b in the neonatal rat. J Neurosci. 2008; 28:12845-12850. [PubMed: 19036978]

Pagliardini S, Janczewski WA, Tan W, Dickson CT, Deisseroth K, Feldman JL. Active expiration induced by excitation of ventral medulla in adult anesthetized rats. J Neurosci. 2011; 31:28952905. [PubMed: 21414911]

Ramanantsoa N, Hirsch MR, Thoby-Brisson M, Dubreuil V, Bouvier J, Ruffault PL, Matrot B, Fortin G, Brunet JF, Gallego J, Goridis C. Breathing without $\mathrm{CO}_{2}$ chemosensitivity in Conditional Phox2b Mutants. J Neurosci. 2011; 31:12880-12888. [PubMed: 21900566]

Rudzinski E, Kapur RP. immunolocalization of the candidate human retrotrapezoid nucleus. Pediatr Dev Pathol. 2010; 13:291-299. [PubMed: 19888871]

Smith JC, Morrison DE, Ellenberger HH, Otto MR, Feldman JL. Brainstem projections to the major respiratory neuron populations in the medulla of the cat. J Comp Neurol. 1989; 281:69-96. [PubMed: 2466879]

Smith JC, Abdala AP, Rybak IA, Paton JF. Structural and functional architecture of respiratory networks in the mammalian brainstem. Philos Trans R Soc Lond B Biol Sci. 2009; 364:25772587. [PubMed: 19651658]

Stornetta RL, Moreira TS, Takakura AC, Kang BJ, Chang DA, West GH, Brunet JF, Mulkey DK, Bayliss DA, Guyenet PG. Expression of Phox $2 b$ by brainstem neurons involved in chemosensory integration in the adult rat. J Neurosci. 2006; 26:10305-10314. [PubMed: 17021186] 
Takakura AC, Moreira TS, Colombari E, West GH, Stornetta RL, Guyenet PG. Peripheral chemoreceptor inputs to retrotrapezoid nucleus (RTN) $\mathrm{CO}_{2}$-sensitive neurons in rats. J Physiol. 2006; 572:503-523. [PubMed: 16455687]

Takakura AC, Moreira TS, Stornetta RL, West GH, Gwilt JM, Guyenet PG. Selective lesion of retrotrapezoid Phox2b-expressing neurons raises the apnoeic threshold in rats. J Physiol. 2008; 586:2975-2991. [PubMed: 18440993]

Thoby-Brisson M, Karlen M, Wu N, Charnay P, Champagnat J, Fortin G. Genetic identification of an embryonic parafacial oscillator coupling to the preBotzinger complex. Nat Neurosci. 2009; 12:1028-1035. [PubMed: 19578380]

Weese-Mayer DE, Berry-Kravis EM, Ceccherini I, Keens TG, Loghmanee DA, Trang H. An official ATS clinical policy statement: congenital central hypoventilation syndrome: genetic basis, diagnosis, and management. Am J Respir Crit Care Med. 2010; 181:626-644. [PubMed: 20208042]

Wenker IC, Kreneisz O, Nishiyama A, Mulkey DK. Astrocytes in the retrotrapezoid nucleus sense H + by inhibition of a Kir4.1-Kir5.1-like current and may contribute to chemoreception by a purinergic mechanism. J Neurophysiol. 2010; 104:3042-3052. [PubMed: 20926613] 


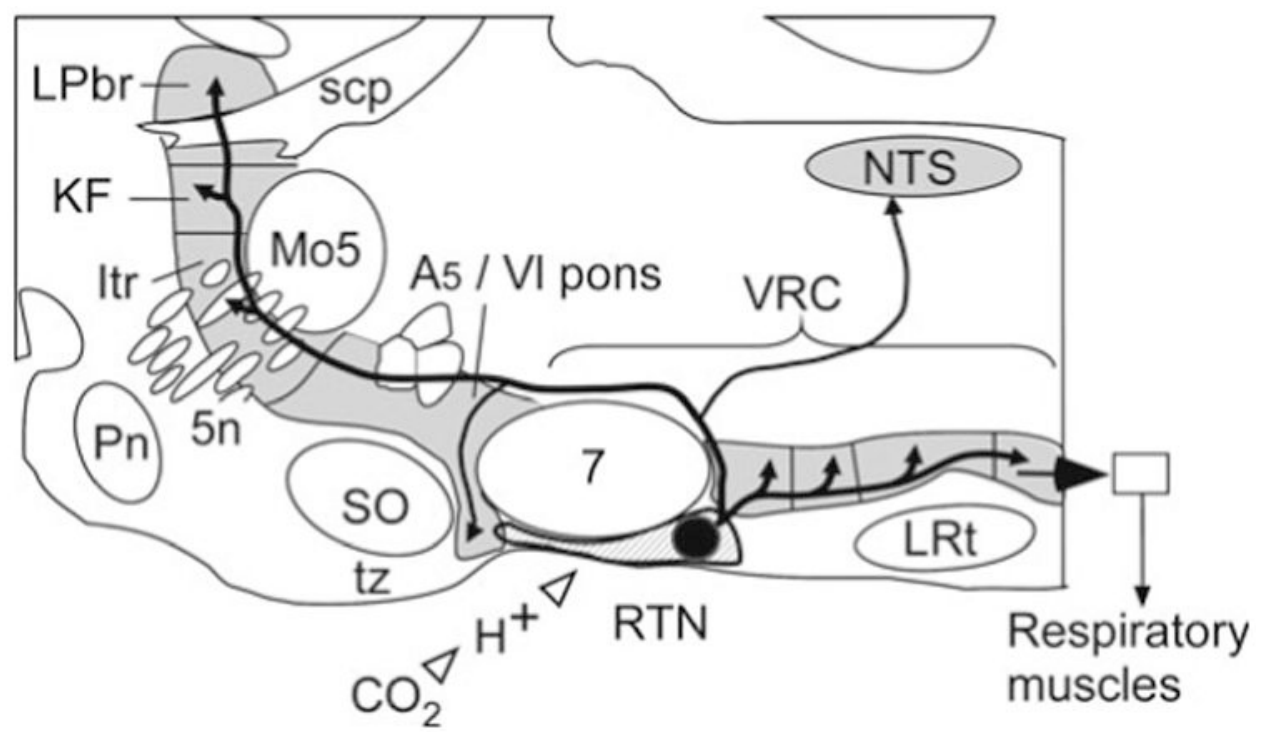

Fig. 16.1.

Location and projection of RTN Phox2b-VGLUT2 neurons. Parasagittal schematic section of the lower brainstem of a rodent. The retrotrapezoid nucleus resides at the ventral surface of the medulla oblongata under the facial motor nucleus (7). RTN innervates all four subdivisions of the ventral respiratory column (VRC), the dorsolateral pons (KF KöllikerFuse nuc., Itr intertrigeminal region, $L P b r$ lateral parabrachial nuc.), the nucleus of the solitary tract (NTS) and the A5 region. Other abbreviations: LRt lateral reticular nucleus, $M o 5$ trigeminal motor nucleus, $P n$ pontine nuclei, $s c p$ superior cerebellar peduncle, $S O$ superior olive, $t z$ trapezoid body, $5 n$ trigeminal nerve 
a
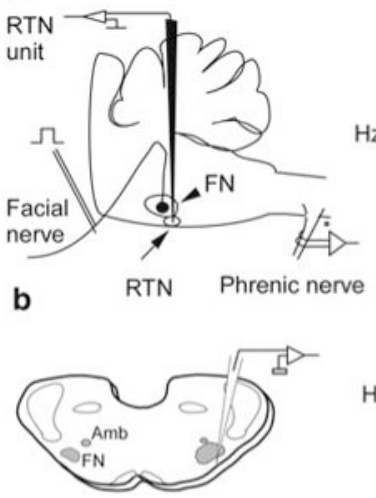
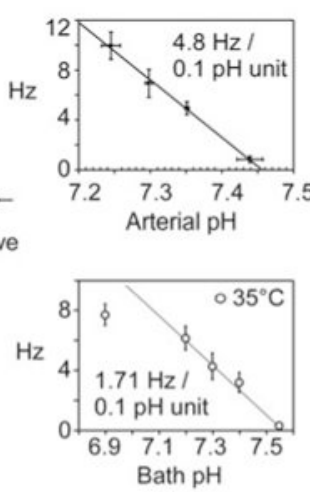
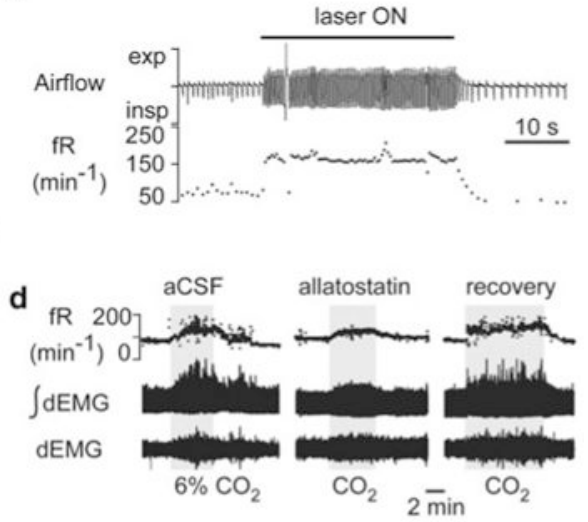

Fig. 16.2.

RTN Phox2b-VGLUT2 neurons and the central respiratory chemoreflex. (a) Left: RTN unit recording in vivo. RTN neurons are found below the facial motor nucleus. The lower boundary of this nucleus is identified by recording antidromic field potentials. Right, relationship between discharge rate of RTN units and arterial $\mathrm{pH}$ in rats in which kynurenic acid was injected icv to block glutamatergic transmission (Source: Guyenet et al. 2005). (b) Left: RTN unit recording in slices from neonatal rodents (FN, facial motor nucleus; Amb, nuc. ambiguus). Right, relationship between discharge rate of RTN units and bath $\mathrm{pH}$.

Although the recordings were made at $35^{\circ} \mathrm{C}$, the $\mathrm{pH}$-sensitivity is only $36 \%$ of its value in vivo (Source: Guyenet et al. 2005). (c) Optogenetic activation of the RTN Phox2b-VGLUT2 neurons elicits massive respiratory stimulation in an awake rat. Channelrhodopsin-2 was selectively transfected into the Phox $2 b$-expressing neurons using a lentiviral vector that expresses its transgene under the control of the artificial promoter PRSx8 (Source: Kanbar et al. 2010). (d) Attenuation of the chemoreflex following selective inhibition of the RTN Phox2b-VGLUT2 neurons. The allatostatin receptor was selectively transfected into the Phox $2 b$-expressing neurons using a lentiviral vector that expresses its transgene under the control of the artificial promoter PRSx8 (Source: Marina et al. 2010). In c and d, the respiratory effects could also be partially due to activation (in c) or inhibition (in d) of the nearby $\mathrm{C} 1$ neurons which also contain Phox $2 \mathrm{~b}$ and therefore also expressed the transgene 


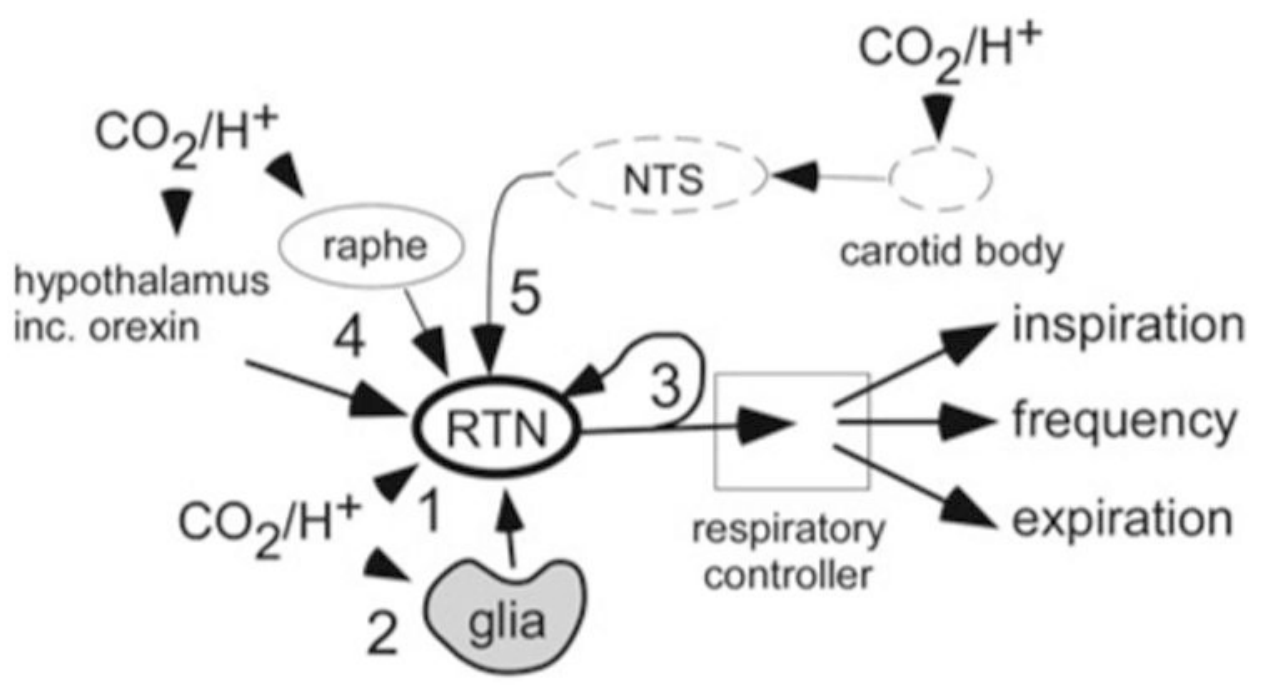

Fig. 16.3.

Five mechanisms may cooperate to produce the high sensitivity of RTN Phox2b-VGLUT2 neurons to hypercapnia. Mechanism 1: RTN neurons respond to acidification through an unidentified cell autonomous response that persists in presence of blockers of glutamate and purinergic transmission in slices (Mulkey et al. 2004). Mechanism 2: ventral surface glial cells are depolarized by acidification and probably contribute to the $\mathrm{CO}_{2}$ sensitivity of the RTN neurons. ATP is the presumed mediator of this paracrine mechanism (Gourine et al. 2010). Mechanism 3: coupling between RTN neurons via gap junctions or recurrent collaterals (depicted) may amplify the cell autonomous effect of $\mathrm{pH}$ on RTN neurons (Fortin and Thoby-Brisson 2009). Mechanism 4: RTN neurons receive input from orexinergic or serotonergic neurons. Subsets of these neurons may respond to changes in brain $\mathrm{pH} / \mathrm{pCO}_{2}$ (Guyenet et al. 2010). Mechanism 5: RTN neurons receive a polysynaptic excitatory input from the carotid bodies (Takakura et al. 2006) 